\title{
Frequency dependent behavior of silicone slide-ring elastomers
}

Tran, Jakob-Anhtu; Madsen, Jeppe; Skov, Anne Ladegaard

Published in:

Electroactive Polymer Actuators and Devices (EAPAD) XXIII

Link to article, DOI:

$10.1117 / 12.2581744$

Publication date:

2021

Document Version

Publisher's PDF, also known as Version of record

Link back to DTU Orbit

Citation (APA):

Tran, J-A., Madsen, J., \& Skov, A. L. (2021). Frequency dependent behavior of silicone slide-ring elastomers. In I. A. Anderson, H. R. Shea, \& J. D. W. Madden (Eds.), Electroactive Polymer Actuators and Devices (EAPAD) XXIII (Vol. 11587). [1158716] SPIE - International Society for Optical Engineering. https://doi.org/10.1117/12.2581744

\section{General rights}

Copyright and moral rights for the publications made accessible in the public portal are retained by the authors and/or other copyright owners and it is a condition of accessing publications that users recognise and abide by the legal requirements associated with these rights.

- Users may download and print one copy of any publication from the public portal for the purpose of private study or research.

- You may not further distribute the material or use it for any profit-making activity or commercial gain

- You may freely distribute the URL identifying the publication in the public portal 


\section{Frequency dependent behavior of silicone slide-ring elastomers}

Tran, Jakob-Anhtu, Madsen, Jeppe, Ladegaard Skov, Anne

Jakob-Anhtu Tran, Jeppe Madsen, Anne Ladegaard Skov, "Frequency dependent behavior of silicone slide-ring elastomers," Proc. SPIE 11587, Electroactive Polymer Actuators and Devices (EAPAD) XXIII, 1158716 (22 March 2021); doi: 10.1117/12.2581744

SPIE. Event: SPIE Smart Structures + Nondestructive Evaluation, 2021, Online Only 


\title{
Frequency dependent behavior of silicone slide-ring elastomers Jakob-Anhtu Tran ${ }^{1}$, Jeppe Madsen ${ }^{1}$, Anne Ladegaard Skov ${ }^{1}$ \\ ${ }^{1}$ Danish Polymer Center, Department of Chemical and Biochemical Engineering, Technical University of Denmark, Søltofts Plads, Building 227, 2800 Kgs. Lyngby, Denmark
}

\begin{abstract}
Slide-ring elastomers consist of mobile cross-links that can rearrange themselves within the network in contrast to conventional elastomers with fixed junctures. This unique feature affects the macroscopic mechanical properties of the sliding elastomers by imparting a distinct sliding elasticity that is caused by the distribution entropy of the sliding cross links. Slide-ring silicone elastomers exhibit two distinct time dependent elastic responses that can be credited to the conformational entropy of the polysiloxane chains and the distribution entropy of the threaded rings. In this work, the transition between rubber elasticity of the silicone chains and the sliding elasticity of the rings has been observed through linear viscoelastic studies. The extensional properties of the elastomers further corroborated the presence of two distinct time dependent viscoelastic profiles. This novel network structure presents the potential to design more intricate dielectric elastomer transducers with two distinctive modes of behavior determined by the operational speed of the system.
\end{abstract}

Keywords: Silicone, slide-ring elastomer, DEA, polyrotaxane, sliding transition

\section{INTRODUCTION}

Slide-ring materials present a unique possibility to produce dynamic networks that can dissipate stress by rearranging their mobile cross-links on a molecular scale while retaining a high degree of elasticity. This phenomenon is caused by the ability for the threaded ring-shaped cross-links to slide on the linear axis chains in a manner similar to a pulley on a zipline (as shown in Figure 1). The highly extensible nature and inherent softness of sliding networks has been attributed to this ability to evenly distribute internal stress when deformed. ${ }^{1}$ The improved elasticity of these networks when compared to traditional fixed systems originates from the distribution entropy of the threaded rings and the possibility to easily revert to their relaxed state through ring sliding. ${ }^{2}$ Slide-ring elastomers have proven to be peculiar in their intrinsic softness, high deformability and toughness, which makes them ideal candidates for their use as dielectric elastomer actuators (DEAs).

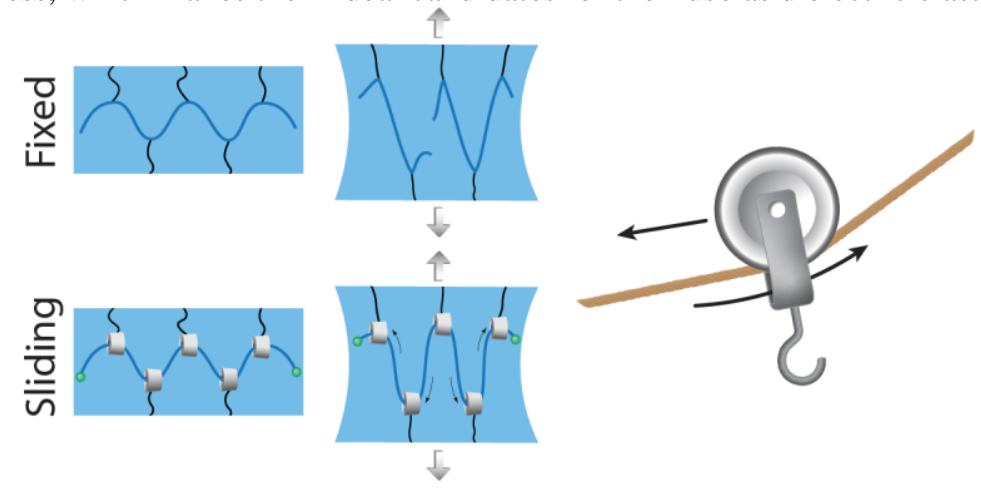

Figure 1: Pulley effect in sliding networks

DEAs have acquired a considerable amount of interest due to the possibilities they present as soft, lightweight, energy transducers with fast response rates. ${ }^{3}$ The applications for this type of innovative actuator range from soft robotics and artificial muscles to self-cleaning surface treatments. ${ }^{4}$ When considering potential materials for DEAs two specific properties are sought after to achieve improved actuation at lower voltages: a high dielectric permittivity and a low Young's modulus. However, tailoring conventional curing formulations for softer elastomers often presents a tradeoff with compromised mechanical integrity and higher viscous loss. ${ }^{3}$ Slide-ring elastomers have thus shown potential to circumvent this issue. 
Sliding cross-linkers based on polyethylene glycol (PEG) and $\alpha$-cyclodextrin $(\alpha-\mathrm{CD})$ have been widely studied due to their commercially available precursors and relative ease of production. This type of cross-linkers have already been utilized for DEA fabrication, but they have so far been limited to poly- $\varepsilon$-caprolactone (PCL) grafted systems, which have a relatively high elastic modulus (around $1 \mathrm{MPa}$ ). ${ }^{5-7}$ The use of alternative side-chain chemistries, such as polysiloxanes, have been restricted by the low reactivity of the cross-linkers and miscibility issues. ${ }^{8}$ These problems are caused by the dense hydrogen bonded structures that form between the threaded cyclodextrin rings and can be solved by chemical modification. ${ }^{9}$

In the current work, we have proposed a synthetic pathway to produce vinyl functional sliding cross-linkers that are soluble in common solvents and can be incorporated in polysiloxane network by means of hydrosilylation. These improvements open up the possibility to prepare sliding silicone elastomers (SSEs) with industrially scalable curing methods. The produced SSEs exhibited a two distinct time dependent stress responses due to the sliding transition of the cross-linkers. This innate characteristic presents the opportunity to design intricate DEAs with discrete modes of behavior that are dependent on the operational speed of the system.

\section{EXPERIMENTAL}

\subsection{Materials}

Polyethylene glycol (PEG 35000) was purchased from Fluka. 1H-Benzotriazol-1-yloxytripyrrolidinophosphonium Hexafluorophosphate (PyBOP) was purchased from Tokyo Chemical Industry Co., Ltd. 1,3-divinyl-1,1,3,3tetramethyldisilazane, platinum-divinyltetramethylsiloxane complex $(3.0 \% \mathrm{Pt})$ as well as hydride and vinyl terminated polydimethylsiloxanes (PDMS $\mathrm{M}_{\mathrm{n}}=20000$ to $24000 \mathrm{~g} \mathrm{~mol}^{-1}$ ) were procured from Gelest Inc. Household bleach (Klorin Original, 5\% available chlorine) was purchased from a local supplier (Salling group A/S). Sylgard ${ }^{\circledR} 184$ elastomer kit was purchased from Dow Chemical Company Ltd. and Ecoflex ${ }^{\mathrm{TM}}$ 00-30 elastomer kit was purchased from FormX Amsterdam. All other chemicals were purchased from Merck $\mathrm{KGaA}$ and used as received unless otherwise stated.

\subsection{Characterization}

${ }^{1} \mathrm{H}-\mathrm{NMR}$ spectra were acquired on a Bruker $300 \mathrm{MHz}$ spectrometer in either deuterated DMSO or chloroform at ambient temperature unless stated otherwise, with the residual solvent used as the internal standard. Size Exclusion Chromatography (SEC) measurements were conducted with Shimadzu instrumentation (SIL-10AD autoinjector, LC10AD liquid chromatograph, RID-10A refractive index detector) with a $10 \mathrm{mM} \mathrm{LiBr}$ DMSO eluent flow of $1.000 \mathrm{~mL} / \mathrm{min}$ at room temperature through a Millipore/Waters Ultrastyragel $10^{5} \AA$ column.

Linear viscoelastic analysis (LVE) was conducted on a TA Discovery HR-1 rheometer with $20 \mathrm{~mm}$ diameter parallel-plate geometry. The measurements were carried out at a fixed strain amplitude of $1 \%$ with a frequency range of 0.01 to $100 \mathrm{~Hz}$. The tensile properties of the elastomers were measured using the Instron 3340 materials testing system (INSTRON, US). Samples of $30 \mathrm{~mm}$ length and $3.18 \mathrm{~mm}$ width were placed between two clamps with an initial separation distance of 20 $\mathrm{mm}$. Strain was defined as $\left(L-L_{0}\right) / L_{0}$ where $L$ is the distance between the clamps during stretching and $L_{0}$ the intial separation distance. The test specimens were elongated uniaxially at a strain rate range of 0.5 to $200 \mathrm{~mm} \mathrm{~min}^{-1}$.

Electrical breakdown tests were performed on an in-house built device based on international standards (IEC 60,243-1 (1998) and IEC 60,243-2 (2001)). A stepwise increase of voltage was applied to the samples (50 V step s${ }^{-1}$ ) at a rate of 0.5 steps $\mathrm{s}^{-1}$. Film thickness was determined through optical microscopy of the film cross-section. Dielectric relaxation spectroscopy (DRS) was performed at room temperature and low electrical field $\left(\sim 1 \mathrm{~V} \mathrm{~mm}^{-1}\right)$ on a Novocontrol Alpha-A high-performance frequency analyser (Concept 40 Top Class System, Novocontrol Technologies GmbH \& Co, Germany) operating in the frequency range $10^{-1}-10^{6} \mathrm{~Hz}$. The samples were approximately $0.1 \mathrm{~mm}$ thick and $20 \mathrm{~mm}$ in diameter.

Actuation measurements were performed by connecting the thin film samples (thickness of 110 to $150 \mu \mathrm{m}$ ) with a high voltage power supply (PS375, Stanford Research Systems Inc.) and applying step-wise increased voltages (500 V per step) until electrical breakdown. The samples were mounted with $20 \%$ pre-strain on a round plastic frame with an inner diameter of $30 \mathrm{~mm}$. Circular electrodes with a diameter of $10 \mathrm{~mm}$ were painted on either sides of the samples with carbon grease. The actuation was determined by video analysis software (Tracker). 


\subsection{Synthesis of polyrotaxanes}

To prepare the carboxylic acid terminated PEG-COOH, ten grams of PEG was oxidized in $100 \mathrm{~mL}$ of DI water with 2,2,6,6-tetramethyl-1-piperidinyloxy radical (TEMPO, $100 \mathrm{mg}, 0.64 \mathrm{mmol}), \mathrm{KBr}(116 \mathrm{mg}, 0.97 \mathrm{mmol})$ and $\mathrm{NaClO}(20$ $\mathrm{mL}, 5 \%$ available chlorine) at a $\mathrm{pH}$ of $11-12$ for $15 \mathrm{~min}$. The oxidation was quenched with $10 \mathrm{~mL}$ of ethanol. The reaction mixture was then acidified with $\mathrm{HCl}$ to a pH below 2. The product was extracted with two $100 \mathrm{~mL}$ aliquots of DCM that were then combined and evaporated in vacuo. The product was dissolved in hot ethanol and precipitated at $-20{ }^{\circ} \mathrm{C}$. The precipitate was then washed with ethanol and dried in vacuo overnight. The end-group conversion was confirmed with ${ }^{1} \mathrm{H}-$ NMR to be $>98 \%$.

3 grams of PEG-COOH and 13.7 grams of $\alpha$-cyclodextrin $(\alpha-\mathrm{CD})$ were dissolved in $100 \mathrm{~mL}$ of DI water and stirred for 15 minutes before overnight complexation at $-5{ }^{\circ} \mathrm{C}$. The complexed pseudo-polyrotaxane (PPR) was then freeze-dried and crushed into a fine powder. The PPR was then end-capped with the addition of 1-adamantylamine (169 mg, $1.1 \mathrm{mmol})$, PyBOP $(581 \mathrm{mg}, 1.1 \mathrm{mmol})$ and $\mathrm{N}, \mathrm{N}$-diisopropylethylamine $(0.2 \mathrm{~mL}, 1.2 \mathrm{mmol})$. The mixture was dissolved in $100 \mathrm{~mL}$ of dry N, N-Dimethylformamide (DMF) and stirred overnight at RT in an inert atmosphere. The reaction mixture was then washed through centrifugation, twice with a 1 to 1 mixture of DMF/methanol and twice with methanol. The obtained solid was then dissolved in dimethylsulfoxide and precipitated in water, followed by repeated washing with water and freezedrying.

\subsection{Chemical modification of polyrotaxanes to obtain vinyl functional slide-ring cross-linkers}

The polyrotaxane $(1 \mathrm{~g}, 7.35 \mu \mathrm{mol})$ was dissolved in dry DMSO $(40 \mathrm{~mL})$ in a nitrogen atmosphere. A premade mixture (4.4 g, $20.4 \mathrm{mmol}$ ) of 1,3-divinyl-1,1,3,3-tetramethyldisilazane (DVTMDS) and 1,1,1,3,3,3-hexamethylsilazane (HMDS) was slowly added to the reaction vessel in a drop-wise manner. The molar ratio of the silylating agents was varied between experiments. The reaction mixture was stirred for 6 hours at $60{ }^{\circ} \mathrm{C}$, followed by addition of dry toluene $(40 \mathrm{~mL})$. The mixture was then left to react for an additional 20 hours. The product was then precipitated in methanol $(800 \mathrm{~mL})$, washed with methanol and dried under vacuum, resulting in a white solid (1.2 g, 69\% yield).

\subsection{Preparation of SSE films}

Hydride terminated PDMS $\left(0.41 \mathrm{~g}, M_{n}=24000 \mathrm{~g} \mathrm{~mol}^{-1}\right)$, vinyl terminated PDMS extenders $\left(0.17 \mathrm{~g}, M_{n}=20000 \mathrm{~g} \mathrm{~mol}^{-1}\right)$ and the slide-ring cross-linkers $\left(0.06 \mathrm{~g}, f_{\text {vinyl }}=0.42 \mathrm{mmol} \mathrm{g}^{-1}\right)$ were dissolved in toluene. Platinum catalyst was added $(1.1 \mathrm{mg}, 50 \mathrm{ppm})$ and the mixture was left to pre-cure for 10 minutes at $80^{\circ} \mathrm{C}$. The mixture was then cast into a Teflon mold and left to evaporate for a day at room temperature before the final overnight curing at $80{ }^{\circ} \mathrm{C}$. Pure PDMS control films were prepared with identical hydride terminated PDMS $\left(1.4 \mathrm{~g}, M_{n}=24000 \mathrm{~g} \mathrm{~mol}^{-1}\right)$ and vinyl terminated PDMS extenders $\left(0.57 \mathrm{~g}, M_{n}=20000 \mathrm{~g} \mathrm{~mol}^{-1}\right)$ and vinyl functional polysiloxane cross-linkers $\left(0.14 \mathrm{~g}, f_{\text {vinyl }}=0.61 \mathrm{mmol} \mathrm{g}^{-1}\right)$. The formulation was mixed on a FlackTek Inc. DAC 150.1 FVZ-K SpeedMixer ${ }^{\mathrm{TM}}$ and then coated on a glass substrate using a film applicator (3540 bird, Elcometer, Germany) with a $200 \mu$ m blade. Commercial Sylgard® 184 and EcoFlex ${ }^{\text {TM }}$ 00-30 silicone films were prepared according to the instructions provided by the suppliers.

\section{RESULTS AND DISCUSSION}

\subsection{Preparing slide-ring silicone elastomers}

The polyrotaxanes consisting of $\alpha$-CD rings threaded on PEG chains were synthesized according to methods described in the literature ${ }^{10}$, with minor modifications. Before the polyrotaxanes could be used as sliding cross-linkers, substitution of the hydroxyl groups on the threaded rings was necessary to increase the compatibility between the polyrotaxanes and silicone and to introduce reactive vinyl functional groups. Chemical modification of the polyrotaxanes is also crucial for improving the solubility of the densely hydrogen-bonded polyrotaxanes. By utilizing two commercially available silylating agents, HMDS and DVTMDS, it was possible to increase the solubility of the cross-linkers while retaining control over the concentration of the introduced reactive groups. The latter was essential in avoiding the formation of fixed junctions on the cyclodextrin rings and ensuring that all of the cross-links were freely mobile in relation to each other (Figure 2). 


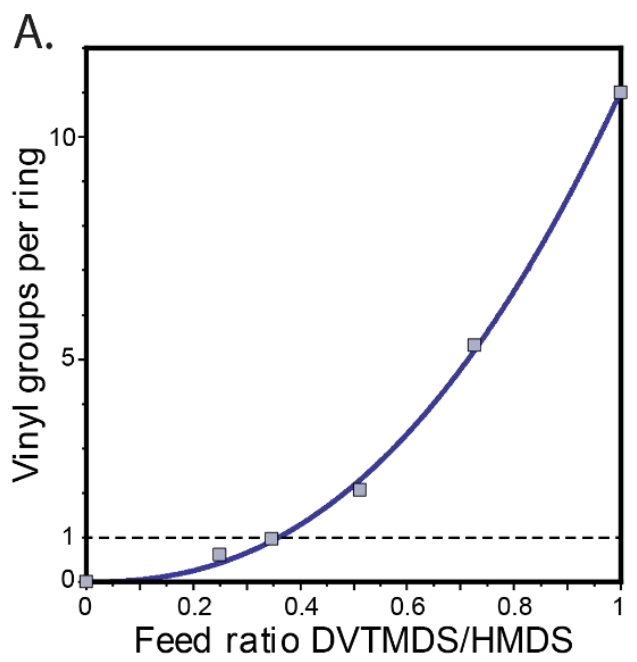

B.
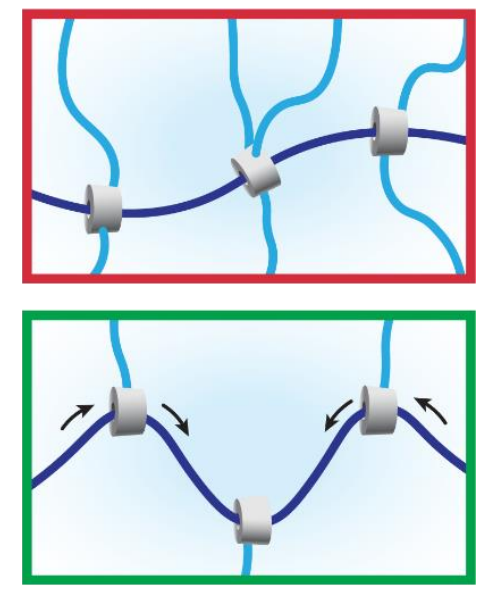

Figure 2: A) Vinyl groups per $\alpha$-CD ring based on the feed ratio B) Comparison of fixed cross-links forming on the $\alpha$-CD rings (top) versus freely sliding networks

The procedure for chemically modifying the polyrotaxanes and cross-linking them with polysiloxane side-chains can be seen in Figure 3. The modified sliding cross-linkers were first pre-cured in toluene with hydride terminated PDMS and chain extenders followed by solvent casting and overnight curing at an elevated temperature.
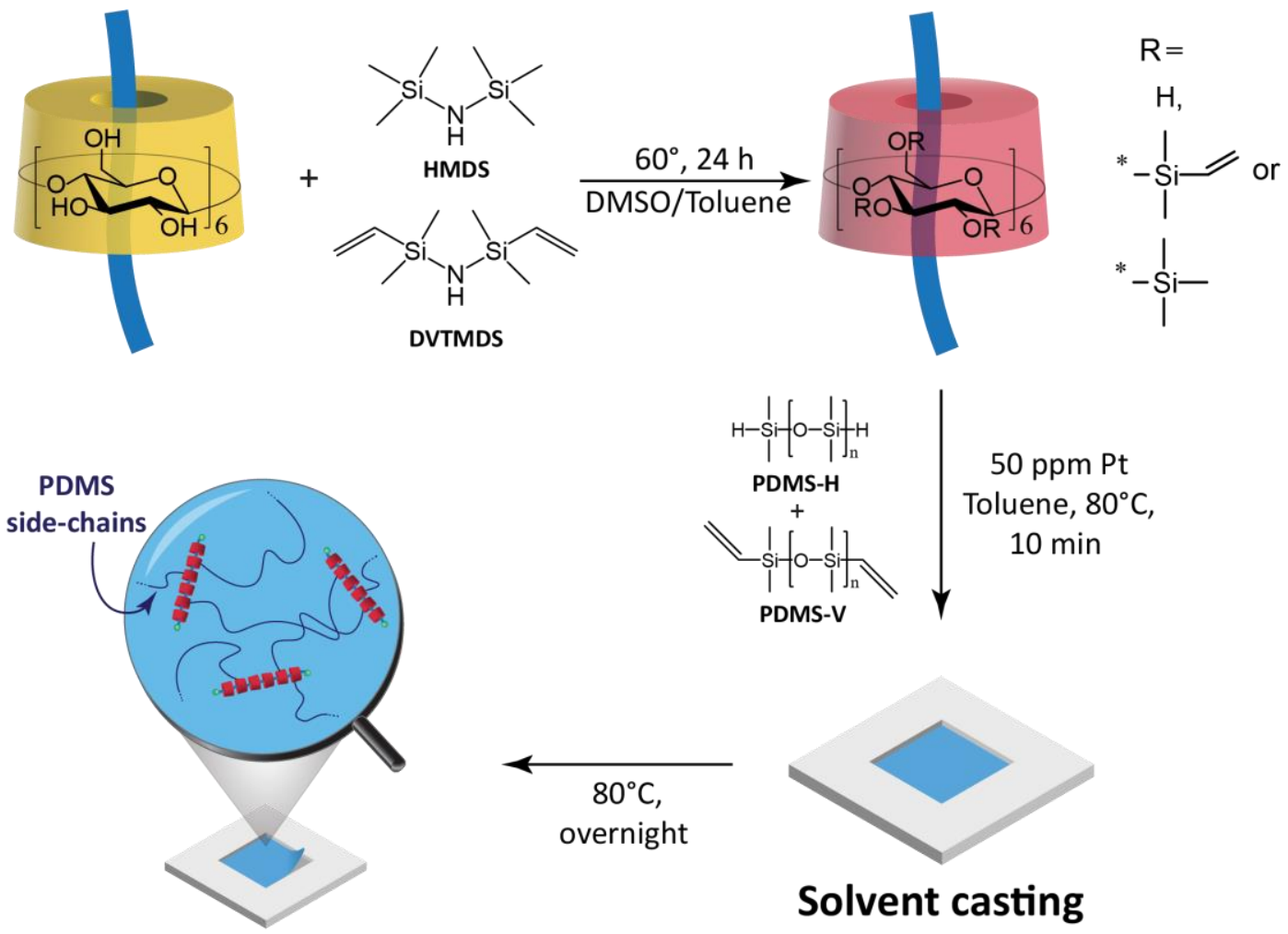

\section{Solvent casting}

Figure 3: Chemical modification of slide-ring cross-linkers and synthesis of slide-ring silicone elastomers

The cured elastomers were transparent, and no visible phase separation could be observed under optical microscopy. A series of sliding elastomers were produced at various cross-linker concentrations determined by the stoichiometric balance, $r$, between the vinyl groups of the cross-linkers and the hydride groups on the terminals of the side-chains. The mechanical and dielectric properties of the samples are presented in Table 1. The elastic modulus $\left(\mathrm{Y}_{5 \%}\right)$ of the elastomers increased in 
relation to the increasing cross-linker concentration. However, the dielectric properties of the samples, such as dielectric permittivity $\left(\varepsilon^{\prime}\right)$ or dielectric loss tangent $(\tan \delta)$, were not affected by the increased polyrotaxane weight fraction. This can be attributed to the change in dielectric properties of the polyrotaxane cross-linkers after chemical modification. Substitution of the polar hydroxyl groups has proved to be effective in lowering both the permittivity and dielectric loss of the polyrotaxanes. It should be noted that the dielectric breakdown strength $\left(E_{b}\right)$ remained relatively stable even for the softer samples with less cross-linkers.

Table 1: Dielectric and mechanical properties of sliding films.

\begin{tabular}{|c|c|c|c|c|c|c|c|}
\hline & $\begin{array}{c}\text { Polyrotaxane } \\
\text { wt } \%\end{array}$ & $\mathbf{r}$ & $\mathbf{Y}_{5 \%} \mathbf{M P a}$ & $\begin{array}{c}\varepsilon^{\prime} @ 0.1 \\
\mathbf{H z}\end{array}$ & $\begin{array}{l}\varepsilon^{\prime} @ 1 \\
\text { MHz }\end{array}$ & $\begin{array}{c}\tan \delta \\
@ 0.1 \mathrm{~Hz}\end{array}$ & $E_{b} V / \mu m$ \\
\hline & 9.4 & 1.5 & 0.529 & 2.7 & 2.8 & $8.8 \cdot 10^{-2}$ & 59.0 \\
\hline & 10.6 & 2.0 & 0.520 & 2.5 & 2.5 & $3.5 \cdot 10^{-2}$ & 65.5 \\
\hline & 14.8 & 2.5 & 2.017 & 2.6 & 2.7 & $1.2 \cdot 10^{-1}$ & 54.6 \\
\hline & 17.3 & 3.0 & 2.962 & 2.8 & 2.8 & $3.2 \cdot 10^{-2}$ & 56.3 \\
\hline & 19.4 & 3.5 & 5.251 & 3.0 & 2.9 & $1.7 \cdot 10^{-2}$ & 49.3 \\
\hline PDMS Control & 0 & 1.5 & 0.192 & 3.3 & 3.3 & $8.0 \cdot 10^{-2}$ & 39.5 \\
\hline Sylgard 184 & 0 & NA & 1.597 & 3.2 & 3.1 & $2.0 \cdot 10^{-2}$ & 113.4 \\
\hline
\end{tabular}

\subsection{Frequency dependent behavior of the silicone slide-ring elastomers}

The rheological properties of the sliding silicone elastomers were studied by linear viscoelastic analysis. A unique transition in the storage modulus could be observed in three of the samples ( $r=1.5$ to 2.5$)$ around the frequency of $0.01 \mathrm{~Hz}$ as seen in Figure 4A. The time-scale of the transition was not affected by the stiffness of the samples indicating that the onset of sliding is determined by the ring entropy within the slide-ring cross-linkers. A similar time-dependent transition between a rubber and a sliding state has been previously observed in slide-ring hydrogels. ${ }^{11}$ To our knowledge, this characteristic sliding transition has not been reported for dry slide-ring elastomers to date. This could be due to the stiffer nature of slide-ring elastomers with PCL grafted side-chains suppressing the magnitude of the transition. The reinforcing effect of the cross-linkers at higher concentrations is also considered to be responsible for the absence of the sliding transition for the two samples with a higher polyrotaxane loading (samples $r=3.0$ and 3.5).
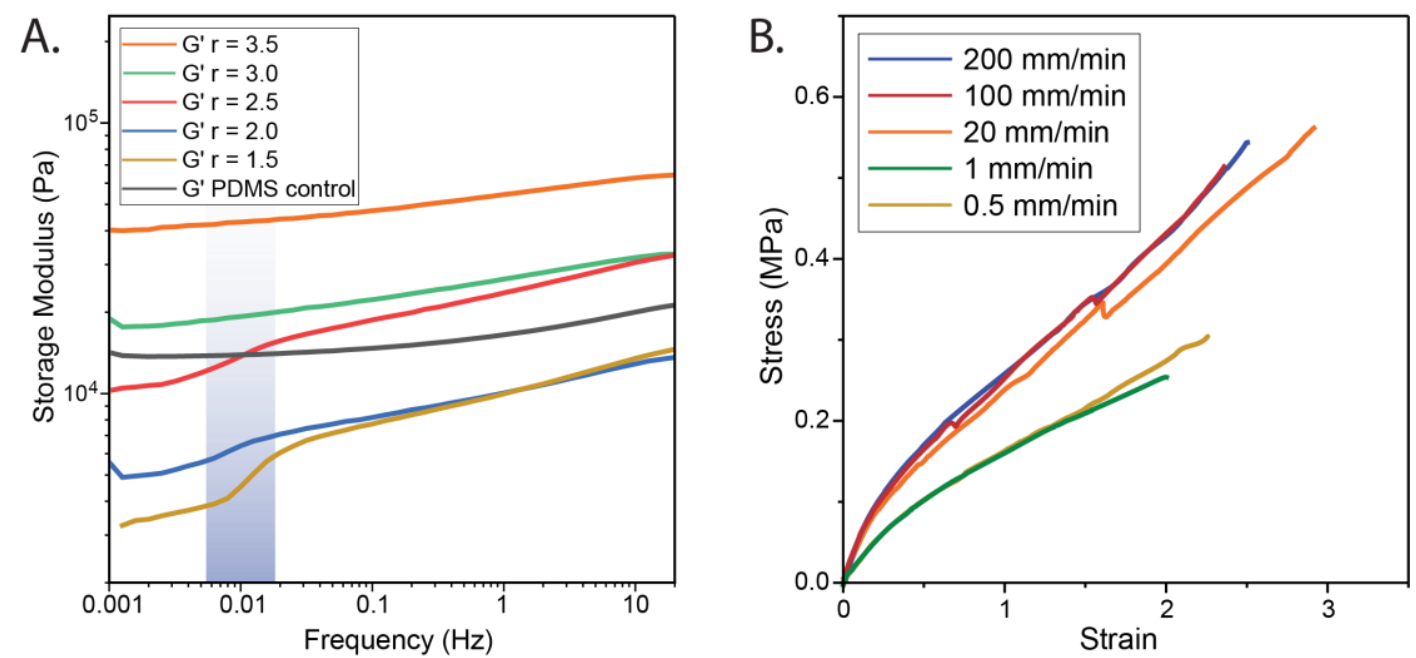

Figure 4: A) Linear viscoelastic study of the sliding elastomers at different concentrations. B) Tensile properties of $r=1.5$

sample at different strain rates $\left(L_{0}=20 \mathrm{~mm}\right)$. All measurements conducted at room temperature

The time dependent nature of the samples was confirmed with extensional rheology (Figure 4B) where two distinct stressstrain responses could be observed depending on the strain rate. The elastic modulus decreases significantly from 0.6 to $0.3 \mathrm{MPa}$ when the strain rate is decreased to $1 \mathrm{~mm} / \mathrm{min}$. In order to compare the time-scale of the extended samples the average Hencky strain rate was determined $\left(\dot{\varepsilon}_{H}=(d L / d t) / L\right)$. The calculations confirmed that the samples with a stiffer 
response were extended at a higher speed $\left(\bar{\varepsilon}_{H}>0.05 \mathrm{sec}^{-1}\right)$ than the transition while the extension rate of the softer samples had crossed the treshold for sliding $\left(\bar{\varepsilon}_{H}=0.004\right.$ and $\left.0.003 \mathrm{sec}^{-1}\right)$.

This dual response presents the potential to design smart DEAs with two distinct modes of operation depending on the actuation speed.

\subsection{Actuation properties of the sliding silicone elastomers}

Preliminary actuation experiments were conducted to compare the novel sliding elastomers with commercial silicone elastomers, Sylgard 184 and Ecoflex 00-30, which are commonly used for fabricating actuators. The relevant material properties of the samples were compiled into a single figure of merit $\left(F_{o m}=3 \varepsilon^{\prime} \varepsilon_{0} E_{b}^{2} / Y\right)^{12}$ to allow for a more direct comparison (as seen in Table 2). The softer sliding actuators exhibited increased actuation when compared to Sylgard 184. The sliding actuators also performed considerably better in regards of breakdown strength when compared to actuators produced with the softer Ecoflex 00-30. These initial results suggest the potential of the slide-ring system as a versatile platform for the preparation of soft actuators with improved cross-linking densities.

Table 2: Dielectric and mechanical properties of sliding films.

\begin{tabular}{lccccc}
\hline & $\mathbf{Y}_{\mathbf{5} \%} \mathbf{M P a}$ & $\boldsymbol{\varepsilon}^{\prime} @ \mathbf{a} \mathbf{0 . 1 H z}$ & $\mathbf{E}_{\mathbf{b}} \mathbf{V} / \mathbf{\mu m}$ & $\begin{array}{c}\text { Actuation strain by } \\
\mathbf{a r e a}(\boldsymbol{\%})\end{array}$ & $\mathbf{F}_{\mathbf{o m}}$ \\
\hline SSE & $0.308^{*}$ & 2.7 & 59.0 & 20.3 & 0.81 \\
Sylgard 184 & 1.597 & 3.2 & 113.4 & 17.5 & 0.68 \\
Ecoflex 00-30 & 0.306 & 3.4 & 42.7 & 21.8 & 0.52
\end{tabular}

*Elastic module of SSE when the strain rate is lower than the inverse sliding transition time

\section{CONCLUSION}

The development of an industrially scalable method to chemically modify polyrotaxane slide-ring cross-linkers allowed for the creation of a new class of sliding silicone elastomers. The sliding elastomers exhibited a peculiar transition between rubber elasticity of the polysiloxane chains and the sliding elasticity of the rings. The extensional properties of the elastomers further corroborated the presence of two distinct time dependent viscoelastic profiles. This novel network structure presents the potential to design more intricate dielectric elastomer transducers with two distinctive modes of behavior determined by the operational speed of the system. 


\section{REFERENCES}

[1] Okumura, Y. and Ito, K., "The Polyrotaxane Gel: A Topological Gel by Figure-of-Eight Cross-links,” Adv. Mater. 13(7), 485-487 (2001).

[2] Kato, K., Okabe, Y., Okazumi, Y. and Ito, K., "A significant impact of host-guest stoichiometry on the extensibility of polyrotaxane gels," Chem. Commun. 51(90), 16180-16183 (2015).

[3] Skov, A. L. and Yu, L., "Optimization Techniques for Improving the Performance of Silicone-Based Dielectric Elastomers," Adv. Eng. Mater. 20(5), 1700762 (2018).

[4] Bauer, S., Bauer-Gogonea, S., Graz, I., Kaltenbrunner, M., Keplinger, C. and Schwödiauer, R., "25th Anniversary Article: A Soft Future: From Robots and Sensor Skin to Energy Harvesters," Adv. Mater. 26(1), 149-162 (2014).

[5] Mayumi, K., Liu, C., Nakai, T., Ishida, M., Takeuchi, H., Inoue, K., Urayama, K., Yokoyama, H. and Ito, K., "Mechanical properties of slide-ring materials for dielectric elastomer actuators," Proc.SPIE 10966 (2019).

[6] Shintake, J., Matsuno, K., Baba, K. and Takeuchi, H., "Characterization of dielectric elastomer actuators made of slide ring materials," Proc.SPIE 10966 (2019).

[7] Zhou, S., Wang, J., Wang, G., Jiang, Z. and Ren, H., "An approach to developing high dielectric constant nanocomposites based on polyrotaxane derivative,” Eur. Polym. J. 90, 312-322 (2017).

[8] Araki, J., Kataoka, T. and Ito, K., "Preparation of a 'sliding graft copolymer', an organic solvent-soluble polyrotaxane containing mobile side chains, and its application for a crosslinked elastomeric supramolecular film," Soft Matter 4(2), 245-249 (2008).

[9] Araki, J. and Ito, K., "Polyrotaxane derivatives. I. Preparation of modified polyrotaxanes with nonionic functional groups and their solubility in organic solvents," J. Polym. Sci. Part A Polym. Chem. 44(21), 6312$6323(2006)$.

[10] Araki, J., Zhao, C. and Ito, K., "Efficient Production of Polyrotaxanes from Cyclodextrin and Poly ( ethylene glycol )," Macromolecules 38(17), 7524-7527 (2005).

[11] Kato, K. and Ito, K., "Dynamic transition between rubber and sliding states attributed to slidable cross-links," Soft Matter 7(19), 8737-8740 (2011).

[12] Sommer-Larsen, P. and Larsen, A. L., "Materials for dielectric elastomer actuators," Electroact. Olymer Actuators Devices 5385, Y. Bar-Cohen, Ed., 68-77 (2004). 